\title{
On the eigenfunctions of the complex Ornstein-Uhlenbeck operators
}

\author{
Yong Chen and Yong Liu
}

\begin{abstract}
Starting from the 1-dimensional complex-valued Ornstein-Uhlenbeck process, we present two natural ways to obtain the associated eigenfunctions of the 2dimensional normal Ornstein-Uhlenbeck operator in the complex Hilbert space $L_{\mathbb{C}}^{2}(\mu)$. We call the eigenfunctions Hermite-Laguerre-Itô polynomials. In addition, the Mehler summation formula for the complex process is shown.
\end{abstract}

\section{Introduction}

There is abundant literature in stochastic analysis concerning the Hermite polynomials and the 1-dimensional real-valued Ornstein-Uhlenbeck process (see [11], [15], [20], and references therein),

$$
\mathrm{d} X_{t}=-b X_{t} \mathrm{~d} t+\sqrt{2 \sigma^{2}} \mathrm{~d} B_{t} .
$$

If the processes $X_{t}, B_{t}$, and the coefficient $b$ are all complex valued, what new findings can we get? Or, say, what is the meaning of the 1-dimensional complexvalued Ornstein-Uhlenbeck process

$$
\mathrm{d} Z_{t}=-\alpha Z_{t} \mathrm{~d} t+\sqrt{2 \sigma^{2}} \mathrm{~d} \zeta_{t},
$$

where $Z_{t}=X_{1}(t)+\mathrm{i} X_{2}(t), \alpha=a e^{\mathrm{i} \theta}=r+\mathrm{i} \Omega$, and $\zeta_{t}=B_{1}(t)+\mathrm{i} B_{2}(t)$ is a complex Brownian motion?

First, it is clear that this complex-valued process can be represented by the 2-dimensional nonsymmetric Ornstein-Uhlenbeck process

$$
\begin{aligned}
{\left[\begin{array}{l}
\mathrm{d} X_{1}(t) \\
\mathrm{d} X_{2}(t)
\end{array}\right] } & =\left[\begin{array}{cc}
-a \cos \theta & a \sin \theta \\
-a \sin \theta & -a \cos \theta
\end{array}\right]\left[\begin{array}{l}
X_{1}(t) \\
X_{2}(t)
\end{array}\right] \mathrm{d} t+\sqrt{2 \sigma^{2}}\left[\begin{array}{l}
\mathrm{d} B_{1}(t) \\
\mathrm{d} B_{2}(t)
\end{array}\right] \\
& =\left[\begin{array}{cc}
-r & \Omega \\
-\Omega & -r
\end{array}\right]\left[\begin{array}{l}
X_{1}(t) \\
X_{2}(t)
\end{array}\right] \mathrm{d} t+\sqrt{2 \sigma^{2}}\left[\begin{array}{l}
\mathrm{d} B_{1}(t) \\
\mathrm{d} B_{2}(t)
\end{array}\right] .
\end{aligned}
$$

From the perspective of physics, (1.2) describes the motion of a charged test particle in the presence of a constant magnetic field and is also called the $A$-Langevin

Kyoto Journal of Mathematics, Vol. 54, No. 3 (2014), 577-596

DOI 10.1215/21562261-2693451, (C) 2014 by Kyoto University

Received December 19, 2012. Revised June 12, 2013. Accepted June 13, 2013.

2010 Mathematics Subject Classification: Primary 60H07; Secondary 60H10, 60G15.

Authors' work partly supported by National Science Foundation of China grants 11071008, 11101137, and 11271029 and by Hunan Provincial NSFC grant 10JJ6014. 
equation in [1, pp. 181-186]. For the process (1.2), its generator is

$$
A=(-r x+\Omega y) \frac{\partial}{\partial x}+(-\Omega x-r y) \frac{\partial}{\partial y}+\sigma^{2}\left(\frac{\partial^{2}}{\partial x^{2}}+\frac{\partial^{2}}{\partial y^{2}}\right),
$$

and its stationary distribution is

$$
\mathrm{d} \mu=\frac{r}{2 \pi \sigma^{2}} \exp \left\{-\frac{r\left(x^{2}+y^{2}\right)}{2 \sigma^{2}}\right\} \mathrm{d} x \mathrm{~d} y .
$$

In the Hilbert space $L^{2}(\mu)$, the adjoint of the operator $A$ is

$$
A^{*}=(-r x-\Omega y) \frac{\partial}{\partial x}-(-\Omega x+r y) \frac{\partial}{\partial y}+\sigma^{2}\left(\frac{\partial^{2}}{\partial x^{2}}+\frac{\partial^{2}}{\partial y^{2}}\right) .
$$

Thus, $A$ is a nonsymmetric operator, and it satisfies $A^{*} A=A A^{*}$ formally (e.g., it is valid for any polynomial), and in fact, one can show that $A$ is a normal operator (see [4]).

Now we address the first question, what are the spectrum and the associated eigenfunctions of the operator $A$ ?

The spectrum and the associated eigenfunctions of the symmetric OrnsteinUhlenbeck operator (i.e., Hermite polynomials) are well known (see [11]). Without the restriction of symmetry, the spectrum of any finite-dimensional OrnsteinUhlenbeck operator in $L^{p}(\mu)$ for $1<p<\infty$ is shown explicitly in [16]. In detail, let $\mathcal{L}=\sum_{i, j=1}^{N} q_{i j} \frac{\partial^{2}}{\partial x_{i} \partial x_{j}}+\sum_{i, j=1}^{N} b_{i j} x_{j} \frac{\partial}{\partial x_{i}}$ be a possibly degenerate OrnsteinUhlenbeck operator, and assume that the associated Markov semigroup has an invariant measure $\mu$; then the spectrum of $\mathcal{L}$ is

$$
\sigma(\mathcal{L})=\left\{\gamma=\sum_{j=1}^{r} n_{j} \lambda_{j}: n_{j} \in \mathbb{N}\right\}
$$

where $\lambda_{1}, \ldots, \lambda_{r}$ are the distinct eigenvalues of the matrix $\left(b_{i j}\right)$ (see [16, Theorem 3.1]). This conclusion has been extended to the infinite-dimensional case in some papers such as [3], [10], [17], and [23]. The spectrum of a hyperbounded Markovian semigroup whose generator is a normal operator on $L^{2}$-space is discussed in [12]. However, for the nonsymmetric Ornstein-Uhlenbeck operator, the associated eigenfunctions are still unknown up until now.

It follows from (1.6) that the spectrum set of the operator $A$ in (1.3) in Hilbert space $L^{2}(\mu)$ is

$$
\sigma(A)=\{-(m+n) r+\mathrm{i}(m-n) \Omega, m, n=0,1,2, \ldots\} .
$$

In the present paper, we will show that eigenfunctions of the operator $A$ are expressed by the Hermite-Laguerre-Itô polynomials (see Definition 2.4 and Theorem 2.2), which are called the Hermite polynomials of complex variables by Itô [8]. Itô used these polynomials to characterize the complex multiple Itô-Wiener integral; that is, his aim was to show a relation similar to the well-known one between the real multiple Itô-Wiener integral and the Hermite polynomials (see [7], [11]). His basic idea was that the Hermite polynomials of complex variables are the coefficients in the expansion of the generating function $\exp (-t \bar{t}+t \bar{z}+\bar{t} z)$ (see [8]). 
This is the complex-valued version of Hermite polynomials as the coefficients in the expansion of the generating function $\exp \left(-t^{2}+2 x t\right)$.

The main purposes of this paper are presenting two different ways to obtain the Hermite-Laguerre-Itô polynomials as eigenfunctions of the operator $A$. One way is that we use the direct and elementary computation by means of the normality of $A$ in $L^{2}(\mu)$. The other way is that by defining the complex variable creation operator and annihilation operator in the complex Hilbert space $L_{\mathbb{C}}^{2}(\mu)$, we verify that Hermite-Laguerre-Itô polynomials can be generated iteratively by the complex creation operator acting on the constant 1 (see Definition 2.4). Those approaches give us some deeper and richer understanding of the 1-dimensional complex-valued Ornstein-Uhlenbeck processes, the Wick product of the symmetric complex Gaussian variable (see Remark 1), and the nonsymmetric stochastic analysis (see [2], [12], [22]). The concrete calculations are given in Section 2.

In Section 3, as applications of the above computations, we obtain the Mehler transform formula and the Mehler summation formula for the complex process (1.1) (or the process (1.2)). In Section 4, by means of a decomposition to the summation of series of up to the 2-dimensional Ornstein-Uhlenbeck operator, we show how to calculate the eigenfunctions of a type of high-dimensional nonsymmetric Ornstein-Uhlenbeck operator. Finally, some tedious computations are listed in the Appendix.

\section{The Hermite-Laguerre-Itô polynomials on $\mathbb{C}$ (or, say, $\mathbb{R}^{2}$ )}

In this section, we present two ways to imply the Hermite-Laguerre-Itô polynomials on $\mathbb{C}$. One is by means of the normality of the operator, and the other is by means of the creation operator and annihilation operator (see [15]).

\subsection{From the perspective of the normal operator}

Let $\rho=\frac{2 \sigma^{2}}{r}$ and $\mathrm{i}=\sqrt{-1}$. Denote by $H_{n}(x, \rho)$ the Hermite polynomials. Let

$$
\mathcal{A}_{s}:=\frac{1}{2}\left(A+A^{*}\right), \quad \mathcal{J}:=\frac{1}{2 \mathrm{i}}\left(A-A^{*}\right) .
$$

Then we have

$$
\mathcal{A}_{s}=\left(\sigma^{2} \frac{\partial^{2}}{\partial x^{2}}-r x \frac{\partial}{\partial x}\right)+\left(\sigma^{2} \frac{\partial^{2}}{\partial y^{2}}-r y \frac{\partial}{\partial y}\right), \quad \mathcal{J}=-\mathrm{i} \Omega\left(y \frac{\partial}{\partial x}-x \frac{\partial}{\partial y}\right) .
$$

It is well known that $\left\{H_{k}\left(x, \frac{\rho}{2}\right) H_{m+n-k}\left(y, \frac{\rho}{2}\right), 0 \leq k \leq m+n\right\}$ is an orthogonal basis of $S_{m+n}$, the characteristic subspace associated with the eigenvalue $-(m+n) r$ of $\mathcal{A}_{s}$. Note that $A A^{*}=A^{*} A$ is valid for any polynomial. Thus $\mathcal{A}_{s} \mathcal{J}=\mathcal{J} \mathcal{A}_{s}$. By restriction of $\mathcal{A}_{s}$ and $\mathcal{J}$ to the finite-dimensional space $S_{m+n}$, the self-adjoint operators $\mathcal{A}_{s}$ and $\mathcal{J}$ have common eigenfunctions which make up an orthogonal basis of $S_{m+n}$. It follows that each common eigenfunction is a linear combination of $H_{k}\left(x, \frac{\rho}{2}\right) H_{m+n-k}\left(y, \frac{\rho}{2}\right), 0 \leq k \leq m+n$. Then we have the following. 
PROPOSITION 2.1

Let $l=m+n$ with $m, n \in \mathbb{N}$ and $\vec{\beta}=\left(\beta_{0}, \beta_{1}, \beta_{2}, \ldots, \beta_{l}\right)^{\prime}$, where $\beta_{k} \in \mathbb{C}$. If the function $J_{m, n}(x, y)=\sum_{k=0}^{l} \beta_{k} H_{k}\left(x, \frac{\rho}{2}\right) H_{l-k}\left(y, \frac{\rho}{2}\right)$ satisfies

$$
\mathcal{J} J_{m, n}(x, y)=-\mathrm{i} \lambda \Omega J_{m, n}(x, y),
$$

then the linear equation

$$
\mathrm{M}(\lambda) \vec{\beta}=0
$$

holds, where $\mathrm{M}(\lambda)$ is an $((l+1) \times(l+1))$ tridiagonal matrix

$$
\mathrm{M}(\lambda)=\left[\begin{array}{cccccccc}
-\lambda & 1 & 0 & 0 & \ldots & 0 & 0 & 0 \\
-l & -\lambda & 2 & 0 & \ldots & 0 & 0 & 0 \\
0 & 1-l & -\lambda & 3 & \ldots & 0 & 0 & 0 \\
\ldots \ldots & \ldots & \ldots & \ldots & \ldots & \ldots & \ldots \ldots & \ldots \\
0 & 0 & 0 & 0 & \ldots & -2 & -\lambda & l \\
0 & 0 & 0 & 0 & \ldots & 0 & -1 & -\lambda
\end{array}\right]
$$

When $\lambda=-(m-n) \mathrm{i}$, (2.4) has a nonzero solution, and the solution $\vec{\beta}$ satisfies

$$
\sum_{k=0}^{l} \beta_{k} \cos ^{k} \theta \sin ^{l-k} \theta=e^{\mathrm{i}(m-n) \theta} c
$$

where $c$ is a constant.

\section{THEOREM 2.2}

The eigenfunction associated with the eigenvalue $-r(m+n)-\mathrm{i}(m-n) \Omega$ of the OU operator $A$ is

$$
J_{m, n}(x, y)= \begin{cases}(-1)^{n} n !(x+\mathrm{i} y)^{m-n} L_{n}^{m-n}\left(x^{2}+y^{2}, \rho\right), & m \geq n, \\ (-1)^{m} m !(x-\mathrm{i} y)^{n-m} L_{m}^{n-m}\left(x^{2}+y^{2}, \rho\right), & m<n,\end{cases}
$$

where $L_{n}^{\alpha}(x, \rho)$ is the Laguerre polynomial (see Definition A.4 or [5], [11], [13], [24]).

Proofs of Proposition 2.1 and Theorem 2.2 are presented in Section A.2.

\subsection{From the perspective of the creation operator and annihilation operator}

An elegant method to deduce the Hermite polynomials is given by means of the creation operator and annihilation operator (see [15]). Let $x \in \mathbb{R}$ and $\mathrm{d} \gamma=$ $\frac{1}{\sqrt{2 \pi \sigma}} e^{-x^{2} / 2 \sigma} \mathrm{d} x$. The operator $\partial$ is the operator of differentiation (or, say, the annihilation operator):

$$
\partial \varphi(x)=\varphi^{\prime}(x), \quad \varphi(x) \in C^{1}(\mathbb{R}) .
$$

Its adjoint operator in the Hilbert space $L^{2}(\gamma)$ is

$$
\partial^{*} \varphi(x)=-\varphi^{\prime}(x)+\frac{x}{\sigma} \varphi(x) .
$$


Then the Hermite polynomials are defined as the sequence ${ }^{\dagger}$

$$
\begin{aligned}
& H_{0}(x)=1, \\
& H_{n}(x)=\sigma \partial^{*} H_{n-1}(x)=\sigma^{n}\left(\partial^{*}\right)^{n} 1 .
\end{aligned}
$$

Let $z=x+\mathrm{i} y$ and $\mathrm{d} \mu=\frac{1}{\pi \rho} e^{-\frac{x^{2}+y^{2}}{\rho}} \mathrm{d} x \mathrm{~d} y$. We consider the complex Hilbert space $L_{\mathbb{C}}^{2}(\mu)$ associated to the inner product

$$
\langle f, g\rangle=\int_{\mathbb{R}^{2}} f \bar{g} \mu(\mathrm{d} x \mathrm{~d} y) .
$$

Set $C_{0}^{1}\left(\mathbb{R}^{2}\right)$ as the collection of $C^{1}$-functions with compact support. We denote by $\partial, \bar{\partial}$ the operators of differentiation (or, say, the complex annihilation operator)

$$
\begin{aligned}
& (\partial \phi)(z)=\frac{\partial}{\partial z} \phi(z)=\frac{1}{2}\left(\frac{\partial}{\partial x}-\mathrm{i} \frac{\partial}{\partial y}\right) \phi(x, y), \\
& (\bar{\partial} \phi)(z)=\frac{\partial}{\partial \bar{z}} \phi(z)=\frac{1}{2}\left(\frac{\partial}{\partial x}+\mathrm{i} \frac{\partial}{\partial y}\right) \phi(x, y) .
\end{aligned}
$$

By [15, Lemma 2.1], the direct calculation yields the adjoint of the operators $\partial, \bar{\partial}$ as follows.

LEMMA 2.3 (COMPLEX CREATION OPERATOR)

Denote by $\partial^{*}, \bar{\partial}^{*}$ the operator defined, for $\phi \in C_{0}^{1}\left(\mathbb{R}^{2}\right)$, by

$$
\left(\partial^{*} \phi\right)(z)=-\frac{\partial}{\partial \bar{z}} \phi(z)+\frac{z}{\rho} \phi(z), \quad\left(\bar{\partial}^{*} \phi\right)(z)=-\frac{\partial}{\partial z} \phi(z)+\frac{\bar{z}}{\rho} \phi(z) .
$$

Then if $\partial \phi, \partial^{*} \psi, \bar{\partial} \phi$, and $\bar{\partial}^{*} \psi \in L_{\mathbb{C}}^{2}(\mu)$ we have

$$
\langle\partial \phi, \psi\rangle=\left\langle\phi, \partial^{*} \psi\right\rangle, \quad\langle\bar{\partial} \phi, \psi\rangle=\left\langle\phi, \bar{\partial}^{*} \psi\right\rangle .
$$

Clearly, $\partial$ commutes with $\bar{\partial}$ and $\partial^{*}$ with $\bar{\partial}^{*}$.

DEFINITION 2.4 (DEFINITION OF THE HERMITE-LAGUERRE-ITÔ POLYNOMIALS)

Let $m, n \in \mathbb{N}$. We define the sequence on $\mathbb{C}$ (or, say, $\mathbb{R}^{2}$ ):

$$
\begin{aligned}
J_{0,0}(z, \rho) & =1, \\
J_{m, n}(z, \rho) & =\rho^{m+n}\left(\partial^{*}\right)^{m}\left(\bar{\partial}^{*}\right)^{n} 1 .
\end{aligned}
$$

We call it the Hermite-Laguerre-Itô polynomials in the present paper.

By induction on $m, n$, we see that $J_{m, n}$ is a polynomial of degree $m+n$ and its term of highest degree is $z^{m} \bar{z}^{n}$. The first few Hermite-Laguerre-Itô polynomials are

$$
\begin{aligned}
& J_{m, 0}=z^{m}, \quad J_{0, n}=\bar{z}^{n}, \\
& J_{1,1}=|z|^{2}-\rho, \quad J_{2,1}=z\left(|z|^{2}-2 \rho\right), \quad J_{3,1}=z^{2}\left(|z|^{2}-3 \rho\right), \quad \ldots
\end{aligned}
$$

$\dagger$ In [15], the variance is $\sigma=1$. 


$$
\begin{aligned}
& J_{1,2}=\bar{z}\left(|z|^{2}-2 \rho\right), \quad J_{2,2}=|z|^{4}-4 \rho|z|^{2}+2 \rho^{2}, \\
& J_{3,2}=z\left(|z|^{4}-6 \rho|z|^{2}+6 \rho^{2}\right), \quad \ldots
\end{aligned}
$$

In general, the following holds.

\section{THEOREM 2.5}

The Hermite-Laguerre-Itô polynomials satisfy

$$
J_{m, n}(z, \rho)=\sum_{r=0}^{m \wedge n}(-1)^{r} r !\left(\begin{array}{c}
m \\
r
\end{array}\right)\left(\begin{array}{l}
n \\
r
\end{array}\right) z^{m-r} \bar{z}^{n-r} \rho^{r} .
$$

The proof of Theorem 2.5 is presented in Section A.3.

\section{REMARK 1}

If $\rho=1$, then $J_{m, n}(z, 1)$ is called the Hermite polynomial of complex variables by Itô, who has shown that a close relation exists between the polynomials and the complex multiple Itô-Wiener integral (see [8]); the reader can also refer to Section A.4. If $\zeta$ is a symmetric complex Gaussian variable, by calculating Feynman diagrams (see [9, p. 131]), the Wick product is represented by

$$
: \zeta^{m} \bar{\zeta}^{n}:=J_{m, n}\left(\zeta, \mathbb{E}|\zeta|^{2}\right)
$$

From the above power series expression, we get that

$$
\begin{aligned}
J_{m, n}(z, \rho) & = \begin{cases}z^{m-n} \sum_{r=0}^{n}(-1)^{r} r !\left(\begin{array}{c}
m \\
r
\end{array}\right)\left(\begin{array}{l}
n \\
r
\end{array}\right)|z|^{2(n-r)} \rho^{r}, & m \geq n, \\
\bar{z}^{n-m} \sum_{r=0}^{m}(-1)^{r} r !\left(\begin{array}{c}
m \\
r
\end{array}\right)\left(\begin{array}{c}
n \\
r
\end{array}\right)|z|^{2(m-r)} \rho^{r}, & m<n,\end{cases} \\
& = \begin{cases}z^{m-n}(-1)^{n} n ! L_{n}^{m-n}\left(|z|^{2}, \rho\right), & m \geq n, \\
\bar{z}^{n-m}(-1)^{m} m ! L_{m}^{n-m}\left(|z|^{2}, \rho\right), & m<n .\end{cases}
\end{aligned}
$$

Thus, we name $J_{m, n}(z, \rho)$ as the Hermite-Laguerre-Itô polynomial in the present paper.

\section{THEOREM 2.6}

The Hermite-Laguerre-Itô polynomials satisfy the following.

(1) Orthonormal basis: $\left\{\left(m ! n ! \rho^{m+n}\right)^{-\frac{1}{2}} J_{m, n}(z, \rho): m, n \in \mathbb{N}\right\}$ is an orthonormal basis of $L_{\mathbb{C}}^{2}(\mu)$.

(2) Eigenfunctions: Let $c \in \mathbb{R}$; then

$$
\begin{aligned}
& {\left[(1+\mathrm{i} c) z \frac{\partial}{\partial z}+(1-\mathrm{i} c) \bar{z} \frac{\partial}{\partial \bar{z}}-2 \rho \frac{\partial^{2}}{\partial z \partial \bar{z}}\right] J_{m, n}(z, \rho)} \\
& \quad=[m+n+\mathrm{i}(m-n) c] J_{m, n}(z, \rho) .
\end{aligned}
$$


(3) Generating function: Let $\lambda \in \mathbb{C}$; then

$$
\exp \left\{\lambda \bar{z}+\bar{\lambda} z-\rho|\lambda|^{2}\right\}=\sum_{m=0}^{\infty} \sum_{n=0}^{\infty} \frac{\bar{\lambda}^{m} \lambda^{n}}{m ! n !} J_{m, n}(z, \rho) .
$$

The proof of Theorem 2.6 is presented in Section A.3.

Let $\rho=\frac{2 \sigma^{2}}{r}, c=\frac{\Omega}{r}$. It is well known that $\frac{\partial}{\partial z}=\frac{1}{2}\left(\frac{\partial}{\partial x}-\mathrm{i} \frac{\partial}{\partial y}\right), \frac{\partial}{\partial \bar{z}}=\frac{1}{2}\left(\frac{\partial}{\partial x}+\right.$ $\left.\mathrm{i} \frac{\partial}{\partial y}\right), 4 \frac{\partial^{2}}{\partial z \partial \bar{z}}=\frac{\partial^{2}}{\partial x^{2}}+\frac{\partial^{2}}{\partial y^{2}} ;$ thus

$$
\begin{aligned}
A & =(-r x+\Omega y) \frac{\partial}{\partial x}+(-\Omega x-r y) \frac{\partial}{\partial y}+\sigma^{2}\left(\frac{\partial^{2}}{\partial x^{2}}+\frac{\partial^{2}}{\partial y^{2}}\right) \\
& =-r\left[(1+\mathrm{i} c) z \frac{\partial}{\partial z}+(1-\mathrm{i} c) \bar{z} \frac{\partial}{\partial \bar{z}}-2 \rho \frac{\partial^{2}}{\partial z \partial \bar{z}}\right] .
\end{aligned}
$$

Then it follows from Theorem 2.6 that we have the following theorem.

\section{THEOREM 2.7}

Let $\rho=\frac{2 \sigma^{2}}{r}$. The Hermite-Laguerre-Itô polynomials $J_{m, n}(z, \rho)$ are the eigenfunctions of 2-dimensional normal Ornstein-Uhlenbeck operators $A$ on $L_{\mathbb{C}}^{2}(\mu)$ with respect to the eigenvalue $-(m+n) r-\mathrm{i}(m-n) \Omega, m, n \geq 0$. And the operator $A$ on the Hilbert space $L_{\mathbb{C}}^{2}(\mu)$ has a pure point spectrum. ${ }^{\dagger}$

These eigenfunctions $J_{m, n}(z, \rho)$ can be employed for, say, orthogonal decomposition like the Hermite polynomials.

\section{COROLLARY 2.8}

Every function $f$ in $L_{\mathbb{C}}^{2}(\mu)$ has a unique series expression

$$
f(x, y)=\sum_{m=0}^{\infty} \sum_{n=0}^{\infty} a_{m, n} \frac{J_{m, n}(z, \rho)}{m ! n ! \rho^{m+n}},
$$

where the coefficients $a_{m, n}$ are given by $a_{m, n}=\left\langle f, J_{m, n}\right\rangle$. Moreover, we have

$$
\|f\|^{2}=\sum_{m=0}^{\infty} \sum_{n=0}^{\infty} \frac{\left|a_{m, n}\right|^{2}}{m ! n ! \rho^{m+n}} .
$$

The following are two examples:

$$
\begin{aligned}
z^{m} \bar{z}^{n} & =\sum_{k=0}^{m \wedge n}\left(\begin{array}{c}
m \\
k
\end{array}\right)\left(\begin{array}{l}
n \\
k
\end{array}\right) k ! \rho^{k} J_{m-k, n-k}(z, \rho), \\
H_{k}\left(x, \frac{\rho}{2}\right) H_{l-k}\left(y, \frac{\rho}{2}\right) & =\frac{\mathrm{i}^{l-k}}{2^{l}} \sum_{m=0}^{l} \sum_{u+v=m}\left(\begin{array}{c}
k \\
u
\end{array}\right)\left(\begin{array}{c}
l-k \\
v
\end{array}\right)(-1)^{v} J_{m, l-m}(z, \rho) .
\end{aligned}
$$

\footnotetext{
$\dagger$ The conclusion of pure point spectrum has been shown in [16] in a different way.
} 
Clearly, the last equation displayed is equivalent to

$$
J_{m, l-m}(z, \rho)=\sum_{k=0}^{l} \mathrm{i}^{l-k} \sum_{u+v=k}\left(\begin{array}{c}
m \\
u
\end{array}\right)\left(\begin{array}{c}
l-m \\
v
\end{array}\right)(-1)^{l-m-v} H_{k}\left(x, \frac{\rho}{2}\right) H_{l-k}\left(y, \frac{\rho}{2}\right),
$$

which is exactly the expression of $J_{m, n}$ given in Proposition 2.1.

\section{The normal Ornstein-Uhlenbeck semigroup and its Mehler summation formula}

As an application to Section 2, we will show the Mehler summation formula for the 2-dimensional normal Ornstein-Uhlenbeck semigroup in this section. This is an analogue of the Mehler summation formula for the real 1-dimensional Ornstein-Uhlenbeck process (see [9, p. 51]); that is, if $\gamma(\mathrm{d} y)=\frac{1}{\sqrt{2 \pi \rho}} e^{-\frac{y^{2}}{2 \rho}} \mathrm{d} y$, we have

$$
\begin{aligned}
\mathcal{M}_{u} \varphi(x) & =\int_{\mathbb{R}} \varphi(y) \frac{1}{\sqrt{1-u^{2}}} \exp \left\{-\frac{u^{2} y^{2}+u^{2} x^{2}-2 u x y}{2 \rho\left(1-u^{2}\right)}\right\} \gamma(\mathrm{d} y), \\
\sum_{n=0}^{\infty} \frac{u^{n}}{n ! \rho^{n}} H_{n}(x, \rho) H_{n}(y, \rho) & =\frac{1}{\sqrt{1-u^{2}}} \exp \left\{-\frac{u^{2} y^{2}+u^{2} x^{2}-2 u x y}{2 \rho\left(1-u^{2}\right)}\right\},
\end{aligned}
$$

where the sum converges pointwise and in $L^{2}(\gamma) \otimes L^{2}(\gamma)$, and $\mathcal{M}_{u}$ is known as the Mehler transform.

Now let $x, y, z \in \mathbb{R}^{2} . A$ is the same as in (2.2). The operator $P_{t}=e^{t A}, t \geq 0$, forms an operator semigroup known as the Ornstein-Uhlenbeck semigroup. It is well known that the semigroup $P_{t}$ has the following explicit representation (see [16]), due to Kolmogorov:

$$
P_{t} \varphi(x)=\frac{1}{\pi \rho\left(1-e^{-2 r t}\right)} \int_{\mathbb{R}^{2}} e^{-\frac{1}{\rho\left(1-e^{-2 r t}\right)}|y|^{2}} \varphi\left(e^{t B} x-y\right) \mathrm{d} y,
$$

where $B=\left[\begin{array}{cc}-r & \Omega \\ -\Omega & -r\end{array}\right]$. Clearly, $e^{t B}=e^{-r t}\left[\begin{array}{cc}\cos \Omega t & \sin \Omega t \\ -\sin \Omega t & \cos \Omega t\end{array}\right]:=e^{-r t} B_{0}(t)$. Thus, a change of variable yields that the normal Mehler formula is

$$
\begin{aligned}
P_{t} \varphi(x) & =\int_{\mathbb{R}^{2}} \varphi\left(e^{-r t} B_{0}(t) x+\sqrt{1-e^{-2 r t}} z\right) \mu(\mathrm{d} z) \\
& =\int_{\mathbb{R}^{2}} \varphi\left(B_{0}(t) x \cos \theta+z \sin \theta\right) \mu(\mathrm{d} z)=P_{t}^{s} \varphi\left(B_{0}(t) x\right),
\end{aligned}
$$

where $\mu$ is as in (1.4), $\cos \theta=e^{-r t}$ with $\theta \in\left(0, \frac{\pi}{2}\right)$, and $P_{t}^{s}$ is the 2-dimensional symmetric Ornstein-Uhlenbeck semigroup associated with the generator $\mathcal{A}_{s}$. The above equation presents a relation between the symmetric and nonsymmetric (normal) Ornstein-Uhlenbeck semigroups.

Denote $u=e^{-r t}, \tilde{B}_{0}(u)=B_{0}\left(-\frac{1}{r} \log u\right)$. A change of variables in (3.1) yields

$$
\begin{aligned}
\mathcal{M}_{u} \varphi(x) & =P_{t} \varphi(x) \\
& =\int_{\mathbb{R}^{2}} \varphi(y) \frac{1}{1-u^{2}} \exp \left\{-\frac{u^{2}|y|^{2}+u^{2}|x|^{2}-2 u\left(\tilde{B}_{0}(u) x, y\right)}{\rho\left(1-u^{2}\right)}\right\} \mu(\mathrm{d} y),
\end{aligned}
$$

where $(x, y)=x^{\prime} y$. 
Equations (2.11) and (2.13) imply that the Mehler transform is characterized by

$$
\mathcal{M}_{u} J_{m, n}=u^{m+n+\mathrm{i}(m-n) c} J_{m, n} .
$$

Since the collection $\left\{J_{m, n}\right\}$ is an orthogonal basis in $L_{\mathbb{C}}^{2}(\mu)$ with $\left\|J_{m, n}\right\|^{2}=$ $m ! n ! \rho^{m+n}$, this means that we also have

$$
\begin{aligned}
& \mathcal{M}_{u} \varphi(x)=\int_{\mathbb{R}^{2}} \sum_{m=0}^{\infty} \sum_{n=0}^{\infty} \frac{u^{m+n+\mathrm{i}(m-n) c}}{m ! n ! \rho^{m+n}} J_{m, n}(x) J_{n, m}(y) \varphi(y) \mu(\mathrm{d} y), \\
& \varphi \in L_{\mathbb{C}}^{2}(\mu),
\end{aligned}
$$

with the sum $\sum \sum \frac{u^{m+n+\mathrm{i}(m-n) c}}{m ! n ! \rho^{m+n}} J_{m, n}(x) J_{n, m}(y)$ converging in $L_{\mathbb{C}}^{2}(\mu) \otimes L_{\mathbb{C}}^{2}(\mu)$ by the Riesz-Fischer theorem. Since the kernels in (3.2) and (3.3) have to coincide, we have the following.

\section{THEOREM 3.1 (MEHLER SUMMATION FORMULA)}

We have

$$
\begin{aligned}
& \sum_{m=0}^{\infty} \sum_{n=0}^{\infty} \frac{u^{m+n+\mathrm{i}(m-n) c}}{m ! n ! \rho^{m+n}} J_{m, n}(x) J_{n, m}(y) \\
& \quad=\frac{1}{1-u^{2}} \exp \left\{-\frac{u^{2}|y|^{2}+u^{2}|x|^{2}-2 u\left(\tilde{B}_{0}(u) x, y\right)}{\rho\left(1-u^{2}\right)}\right\}
\end{aligned}
$$

where the sum converges pointwise and in $L_{\mathbb{C}}^{2}(\mu) \otimes L_{\mathbb{C}}^{2}(\mu)$.

The pointwise convergence can be showed by the same argument as in the proof of (2.12).

\section{The processes are decomposed into some 1-dimensional complex-valued processes}

In this section, suppose that $\mathrm{C}$ is a normal $n$-by- $n$ matrix and the OrnsteinUhlenbeck processes satisfy

$$
\mathrm{d} \vec{X}(t)=C \vec{X}(t) \mathrm{d} t+\sqrt{2 \sigma^{2}} \mathrm{~d} \vec{B}(t) .
$$

Its generator is

$$
\mathcal{A}=\sigma^{2} \Delta_{\vec{x}}+(\mathrm{C} \vec{x}) \cdot \nabla_{\vec{x}}
$$

Clearly, it is an $n$-dimensional normal Ornstein-Uhlenbeck operator.

As in [14] and [16], we do a linear transform according to the canonical form of the normal matrix C. Since $C$ is normal, there is a real orthogonal matrix $Q$ such that

$$
\mathrm{Q}^{\prime} \mathrm{CQ}=\operatorname{diag}\left\{A_{1}, A_{2}, \ldots, A_{l}\right\}
$$


where each $A_{j}$ is either a real 1-by-1 matrix or a real 2-by-2 matrix of the form

$$
A_{j}=\left[\begin{array}{cc}
\alpha_{j} & \beta_{j} \\
-\beta_{j} & \alpha_{j}
\end{array}\right],
$$

where $\alpha_{j} \pm \mathrm{i} \beta_{j}, \beta_{j} \neq 0$, is a pair of conjugate complex eigenvalues of $\mathrm{C}$ (see $[6$, p. 105]). That is to say, its canonical form is a block diagonal matrix. If $\mathrm{C}$ is not symmetric, then there is at least one $A_{j}$ which is a 2 -by-2 matrix. Let $\vec{Y}(t)=$ $\mathrm{Q}^{\prime} \vec{X}_{t}, \vec{y}=\mathrm{Q}^{\prime} \vec{x}$; then $\vec{Y}(t)$ satisfies

$$
\mathrm{d} \vec{Y}(t)=\operatorname{diag}\left\{A_{1}, A_{2}, \ldots, A_{l}\right\} \vec{Y}(t) \mathrm{d} t+\sqrt{2 \sigma^{2}} \mathrm{~d} \vec{B}(t) .
$$

Its generator is

$$
\tilde{\mathcal{A}}=\sigma^{2} \Delta_{\vec{y}}+\left(\operatorname{diag}\left\{A_{1}, A_{2}, \ldots, A_{l}\right\} \vec{y}\right) \cdot \nabla_{\vec{y}} .
$$

The eigenfunctions of the 2-dimensional normal operator (1.3) yield the eigenfunctions of the operator $\tilde{\mathcal{A}}$ (see [19, p. 51]). Note that the eigenfunctions of the operators $\mathcal{A}$ and $\tilde{\mathcal{A}}$ are the same up to an orthogonal transform (i.e., $\mathcal{A} f(\vec{x})=\tilde{\mathcal{A}} g(\vec{y})$ when $\left.f(\vec{x})=g\left(\mathrm{Q}^{\prime} \vec{x}\right)\right)$; thus we can get the eigenfunctions of the operator $\mathcal{A}$. That is to say, we have found a way to calculate all the eigenfunctions of the normal Ornstein-Uhlenbeck operator (4.1). The following is a concrete example.

\section{EXAMPLE 1}

The following equation describes the coupling diffusion on the lattice of the circle:

$$
\mathrm{d} \vec{X}(t)=C \vec{X}(t) \mathrm{d} t-r \vec{X}(t) \mathrm{d} t+\sqrt{2 \sigma^{2}} \mathrm{~d} \vec{B}(t),
$$

where $\mathrm{C}$ is an $n$-by- $n(n \geq 3)$ tridiagonal matrix

$$
\mathrm{C}=\left[\begin{array}{ccccc}
-(a+b) & a & 0 & \cdots & b \\
b & -(a+b) & a & \cdots & 0 \\
\vdots & \vdots & \vdots & \vdots & \vdots \\
0 & 0 & \cdots & -(a+b) & a \\
a & 0 & \cdots & b & -(a+b)
\end{array}\right]
$$

Denote by $\omega_{j}=\exp \left(\mathrm{i} \frac{2 j \pi}{n}\right)$ the $j$ th unit root of 1 , and $\omega_{j}^{*}$ is the complex conjugate of $\omega_{j}$. Clearly, the eigenvectors of $\mathrm{C}$ are

$$
\vec{\varphi}_{j}=\frac{1}{\sqrt{n}}\left(1, \omega_{j}, \omega_{j}^{2}, \ldots, \omega_{j}^{n-1}\right)^{\prime}, \quad j=0,1,2, \ldots, n-1,
$$

and $C$ is a normal matrix. Let $L_{k}=\operatorname{span}\left\{\operatorname{Re}\left(\vec{\varphi}_{k}\right), \operatorname{Im}\left(\vec{\varphi}_{k}\right)\right\}, k=0,1,2, \ldots,\left[\frac{n}{2}\right]$, and denote by $P_{L_{k}}$ the project operator. Then there is a resolution of the identity $\mathrm{Id}=\sum_{k=0}^{\left[\frac{n}{2}\right]} P_{L_{k}}$. Thus

$$
\vec{X}(t)=\sum_{k=0}^{\left[\frac{n}{2}\right]} P_{L_{k}} \vec{X}(t)=\sum_{k=0}^{\left[\frac{n}{2}\right]}\left(Y_{k}(t) \operatorname{Re}\left(\vec{\varphi}_{k}\right)+Z_{k}(t) \operatorname{Im}\left(\vec{\varphi}_{k}\right)\right),
$$


where $\left(Y_{k}(t), Z_{k}(t)\right)$ satisfies the equation

$$
\left[\begin{array}{l}
\mathrm{d} Y_{k}(t) \\
\mathrm{d} Z_{k}(t)
\end{array}\right]=A_{k}\left[\begin{array}{l}
Y_{k}(t) \\
Z_{k}(t)
\end{array}\right] \mathrm{d} t+\sqrt{2 \sigma^{2}}\left[\begin{array}{l}
\mathrm{d} B_{1}(t) \\
\mathrm{d} B_{2}(t)
\end{array}\right]
$$

with

$$
A_{k}=\left[\begin{array}{cc}
-r+(a+b)\left(\cos \frac{2 k \pi}{n}-1\right) & (a-b) \sin \frac{2 k \pi}{n} \\
(b-a) \sin \frac{2 k \pi}{n} & -r+(a+b)\left(\cos \frac{2 k \pi}{n}-1\right)
\end{array}\right] .
$$

\section{Appendix}

\section{A.1 Hermite polynomials and Laguerre polynomials}

To be self-contained, we list the well-known results of the Hermite polynomials and the Laguerre polynomials, for which the reader can refer to the references (see [5], [11], [13], [21], [24]).

\section{DEFINITION A.1}

The Hermite polynomials are defined by the formula

$$
H_{n}(x, \rho)=(-\rho)^{n} e^{x^{2} / 2 \rho} \frac{\mathrm{d}^{n}}{\mathrm{~d} x^{n}} e^{-x^{2} / 2 \rho}, \quad n=1,2, \ldots
$$

Clearly, it has power series expression,

$$
H_{n}(x, \rho)=\sum_{k=0}^{[n / 2]}\left(\begin{array}{c}
n \\
2 k
\end{array}\right)(2 k-1) ! ! x^{n-k}(-\rho)^{k} .
$$

\section{PROPOSITION A.2}

The Hermite polynomials $H_{n}(x, \rho)$ satisfy

(1) partial derivatives: $\frac{\mathrm{d}}{\mathrm{d} x} H_{n}(x, \rho)=n H_{n-1}(x, \rho)$,

(2) recursion formula: $H_{n+1}(x, \rho)=x H_{n}(x, \rho)-n \rho H_{n-1}(x, \rho)$,

(3) orthogonality: $\frac{1}{\sqrt{2 \pi \rho}} \int_{-\infty}^{+\infty} e^{-\frac{x^{2}}{2 \rho}} H_{n}(x, \rho) H_{m}(x, \rho) \mathrm{d} x=n ! \rho^{n} \delta_{n m}$,

(4) integral representation:

$$
H_{n}(x, \rho)=\frac{(-\mathrm{i})^{n}}{\sqrt{2 \pi \rho}} \int_{-\infty}^{\infty} t^{n} e^{-\frac{1}{2 \rho}(t-\mathrm{i} x)^{2}} \mathrm{~d} t, \quad n=0,1,2, \ldots
$$

\section{DEFINITION A.3 (BESSEL'S INTEGRALS)}

The definition of the Bessel function of the first kind, for integer values of $n$, is possible using an integral representation

$$
\mathrm{J}_{n}(x)=\frac{1}{2 \pi} \int_{-\pi}^{\pi} e^{-\mathrm{i}(n \tau-x \sin \tau)} \mathrm{d} \tau
$$

By the periodicity of the triangle function, we have that $\forall \alpha \in \mathbb{R}$,

$$
\mathrm{J}_{n}(x)=\frac{e^{-\mathrm{i} n \alpha}}{2 \pi} \int_{-\pi}^{\pi} e^{\mathrm{i}[x \sin (\alpha-\theta)+n \theta]} \mathrm{d} \theta .
$$




\section{DEFINITION A.4}

The Laguerre polynomials $L_{n}^{\alpha}(x, \rho)$ are defined by the formula (see [13, p. 76])

$$
L_{n}^{\alpha}(x, \rho)=\frac{\rho^{n}}{n !} x^{-\alpha} e^{\frac{x}{\rho}} \frac{\mathrm{d}^{n}}{\mathrm{~d} x^{n}}\left(e^{-\frac{x}{\rho}} x^{n+\alpha}\right), \quad n=1,2, \ldots,
$$

for arbitrary real $\alpha>-1$. Clearly, it has power series expression

$$
L_{n}^{\alpha}(x, \rho)=\frac{(-1)^{n}}{n !} \sum_{r=0}^{n}(-1)^{r} r !\left(\begin{array}{c}
n+\alpha \\
r
\end{array}\right)\left(\begin{array}{c}
n \\
r
\end{array}\right) x^{n-r} \rho^{r} .
$$

\section{PROPOSITION A.5}

The Laguerre polynomials $L_{n}^{\alpha}(x, \rho)$ satisfy

(1) partial derivatives: $\frac{\partial}{\partial x} L_{n}^{\alpha}(x, \rho)=-L_{n-1}^{\alpha+1}(x, \rho)$;

(2) recursion formula:

$$
\begin{aligned}
L_{n}^{\alpha}(x, \rho) & =L_{n}^{\alpha+1}(x, \rho)-\rho L_{n-1}^{\alpha+1}(x, \rho), \\
x \frac{\partial}{\partial x} L_{n}^{\alpha}(x, \rho) & =n L_{n}^{\alpha}(x, \rho)-(n+\alpha) \rho L_{n-1}^{\alpha}(x, \rho) ;
\end{aligned}
$$

(3) orthogonality: $\int_{0}^{+\infty} x^{\alpha} e^{-\frac{x}{\rho}} L_{m}^{\alpha}(x, \rho) L_{n}^{\alpha}(x, \rho) \mathrm{d} x=\rho^{m+n+\alpha+1} \frac{\Gamma(\alpha+n+1)}{n !} \times$ $\delta_{n m}$

(4) integral representation:

$$
\begin{gathered}
L_{n}^{\alpha}(x, \rho)=\frac{e^{\frac{x}{\rho}} x^{-\alpha / 2}}{n ! \rho} \int_{0}^{\infty} t^{n+\frac{1}{2} \alpha} \mathrm{J}_{\alpha}\left(\frac{2}{\rho} \sqrt{x t}\right) e^{-\frac{t}{\rho}} \mathrm{d} t, \\
\alpha>-1, n=0,1,2, \ldots,
\end{gathered}
$$

where $\mathrm{J}_{v}(x)$ is the Bessel function of order $v$.

\section{A.2 Proof of Theorem 2.2}

For simplicity, we denote $H_{n}\left(x, \frac{\rho}{2}\right)$ by $H_{n}(x)$ in this subsection.

Proof of Proposition 2.1

It follows from the recursion relation of Hermite polynomials (see Proposition A.2) that

$$
\begin{aligned}
\frac{\mathrm{i}}{\Omega} & \mathcal{J}\left[H_{k}(x) H_{l-k}(y)\right] \\
& =y H_{l-k}(y) \frac{\mathrm{d}}{\mathrm{d} x} H_{k}(x)-x H_{k}(x) \frac{\mathrm{d}}{\mathrm{d} y} H_{l-k}(y) \\
& =y H_{l-k}(y) k H_{k-1}(x)-x H_{k}(x)(l-k) H_{l-k-1}(y) \\
& =k H_{k-1}(x) H_{l-k+1}(y)-(l-k) H_{k+1}(x) H_{l-k-1}(y) .
\end{aligned}
$$

Since $\frac{\mathrm{i}}{\Omega} \mathcal{J} J_{m, n}(x, y)=\lambda J_{m, n}(x, y)$ and $J_{m, n}(x, y)=\sum_{k=0}^{l} \beta_{k} H_{k}(x) H_{l-k}(y)$, we obtain 


$$
\begin{aligned}
\frac{\mathrm{i}}{\Omega} & \mathcal{J}\left[\sum_{k=0}^{l} \beta_{k} H_{k}(x) H_{l-k}(y)\right] \\
& =\sum_{k=0}^{l} \beta_{k}\left[k H_{k-1}(x) H_{l-k+1}(y)-(l-k) H_{k+1}(x) H_{l-k-1}(y)\right] \\
& =\lambda \sum_{k=0}^{l} \beta_{k} H_{k}(x) H_{l-k}(y) .
\end{aligned}
$$

Let $\beta_{-1}=\beta_{l+1}=0$. Since $H_{k}(x) H_{l-k}(y)$ are orthogonal, we have

$$
-(l-(k-1)) \beta_{k-1}-\lambda \beta_{k}+(k+1) \beta_{k+1}=0 .
$$

It is exactly the linear equation (2.4).

It follows from [18, Problems 373, 399] that

$$
\begin{aligned}
\operatorname{det}\{\mathrm{M}(\lambda)\} & =\left|\begin{array}{cccccccc}
-\lambda & \mathrm{i} & 0 & 0 & \ldots & 0 & 0 & 0 \\
l \mathrm{i} & -\lambda & 2 \mathrm{i} & 0 & \ldots & 0 & 0 & 0 \\
0 & (l-1) \mathrm{i} & -\lambda & 3 \mathrm{i} & \ldots & 0 & 0 & 0 \\
\ldots & \ldots & \ldots & \ldots & \ldots & \ldots & \ldots & \ldots \\
0 & 0 & 0 & 0 & \ldots & 2 \mathrm{i} & -\lambda & l \mathrm{i} \\
0 & 0 & 0 & 0 & \ldots & 0 & \mathrm{i} & -\lambda
\end{array}\right| \\
& =\mathrm{i}^{l+1}\left|\begin{array}{cccccccc}
\lambda \mathrm{i} & 1 & 0 & 0 & \ldots & 0 & 0 & 0 \\
l & \lambda \mathrm{i} & 2 & 0 & \ldots & 0 & 0 & 0 \\
0 & l-1 & \lambda \mathrm{i} & 3 & \ldots & 0 & 0 & 0 \\
\ldots \ldots & \ldots & \ldots & \ldots \\
0 & 0 & 0 & 0 & \ldots & 2 & \lambda \mathrm{i} & l \\
0 & 0 & 0 & 0 & \ldots & 0 & 1 & \lambda \mathrm{i}
\end{array}\right|=\mathrm{i}^{l+1} \prod_{m=0}^{l}(\lambda \mathrm{i}+l-2 m) \\
& =(-1)^{l+1} \prod_{m=0}^{l}(\lambda+(2 m-l) \mathrm{i})=(-1)^{l+1} \prod_{m=0}^{l}(\lambda+(m-n) \mathrm{i}) .
\end{aligned}
$$

Thus when $\lambda=-(m-n)$ i, $(2.4)$ has a nonzero solution. ${ }^{\dagger}$

Set $I(\theta)=\sum_{k=0}^{l} \beta_{k} \cos ^{k} \theta \sin ^{l-k} \theta$. Differentiating $I(\theta)$ yields

$$
\begin{aligned}
I^{\prime}(\theta) & =\sum_{k=0}^{l} \beta_{k}\left[-k \cos ^{k-1} \theta \sin ^{l-k+1} \theta+(l-k) \cos ^{k+1} \theta \sin ^{l-k-1} \theta\right] \\
& =-\sum_{k=0}^{l}\left[(k+1) \beta_{k+1}-(l-(k-1)) \beta_{k-1}\right] \cos ^{k} \theta \sin ^{l-k} \theta \\
& =-\lambda \sum_{k=0}^{l} \beta_{k} \cos ^{k} \theta \sin ^{l-k} \theta \quad(\text { by (A.9)) } \\
& =(m-n) \mathrm{i} I(\theta) .
\end{aligned}
$$

$\dagger$ In fact, we present an alternative way to get (1.7), that is, the spectrum of the operator $A$. 
Then we have that

$$
\sum_{k=0}^{l} \beta_{k} \cos ^{k} \theta \sin ^{l-k} \theta=e^{\mathrm{i}(m-n) \theta} c,
$$

where $c$ is a constant.

Proof of Theorem 2.2

Without loss of generality, we choose $c=1$ in (A.10). We need only to show the case $m \geq n$ :

$$
\begin{aligned}
J_{m, n}(x, y) \\
=\sum_{k=0}^{l} \beta_{k} H_{k}\left(x, \frac{\rho}{2}\right) H_{l-k}\left(y, \frac{\rho}{2}\right) \\
=\sum_{k=0}^{l} \beta_{k}\left[\frac{(-\mathrm{i})^{l}}{\pi \rho} \iint_{\mathbb{R}^{2}} t^{k} s^{l-k} e^{-\frac{1}{\rho}(t-\mathrm{i} x)^{2}} e^{-\frac{1}{\rho}(s-\mathrm{i} y)^{2}} \mathrm{~d} t \mathrm{~d} s\right] \quad(\text { by }(\mathrm{A} .2)) \\
=\frac{(-\mathrm{i})^{l}}{\pi \rho} e^{\frac{1}{\rho}\left(x^{2}+y^{2}\right)} \\
\quad \times \int_{0}^{\infty} e^{-\frac{1}{\rho} r^{2}} r^{l+1} \mathrm{~d} r \int_{-\pi}^{\pi}\left[\sum_{k=0}^{l} \beta_{k} \cos ^{k} \theta \sin ^{l-k} \theta\right] e^{\frac{2 \mathrm{i} r}{\rho}(x \cos \theta+y \sin \theta)} \mathrm{d} \theta
\end{aligned}
$$

(by polar coordinate transformation of $t, s$ )

$$
\begin{aligned}
= & \frac{(-\mathrm{i})^{l}}{\pi \rho} e^{\frac{1}{\rho}\left(x^{2}+y^{2}\right)} \\
& \times \int_{0}^{\infty} e^{-\frac{1}{\rho} r^{2}} r^{l+1} \mathrm{~d} r \int_{-\pi}^{\pi} e^{\frac{2 \mathrm{i} r}{\rho}(x \cos \theta+y \sin \theta)+\mathrm{i}(m-n) \theta} \mathrm{d} \theta \quad(\text { by }(\mathrm{A} .10)) \\
= & \frac{(-\mathrm{i})^{l}}{\pi \rho} e^{\frac{1}{\rho}\left(x^{2}+y^{2}\right)} \int_{0}^{\infty} e^{-\frac{1}{\rho} r^{2}} r^{l+1} \mathrm{~d} r \int_{-\pi}^{\pi} e^{\mathrm{i}\left[\frac{2 r}{\rho} \sqrt{x^{2}+y^{2}} \sin (\alpha-\theta)+(m-n) \theta\right]} \mathrm{d} \theta
\end{aligned}
$$

(by polar coordinate transformation of $-y, x$, i.e., $-y+\mathrm{i} x=\sqrt{x^{2}+y^{2}} e^{\mathrm{i} \alpha}$ )

$$
\begin{aligned}
= & \frac{(-\mathrm{i})^{l}}{\rho} e^{\mathrm{i}(m-n) \alpha+\frac{1}{\rho}\left(x^{2}+y^{2}\right)} \\
& \times \int_{0}^{\infty} r^{l} \mathrm{~J}_{m-n}\left(\frac{2 r}{\rho} \sqrt{\left(x^{2}+y^{2}\right)}\right) e^{-\frac{1}{\rho} r^{2}} 2 r \mathrm{~d} r \quad(\text { by }(\text { A.3 })) \\
= & \frac{(-\mathrm{i})^{l}}{\rho} e^{\mathrm{i}(m-n) \alpha+\frac{1}{\rho}\left(x^{2}+y^{2}\right)} \\
& \times \int_{0}^{\infty} \gamma^{n+\frac{m-n}{2}} \mathrm{~J}_{m-n}\left(\frac{2}{\rho} \sqrt{\left(x^{2}+y^{2}\right) \gamma}\right) e^{-\frac{\gamma}{\rho}} \mathrm{d} \gamma \quad\left(\text { let } \gamma=r^{2}\right) \\
= & (-\mathrm{i})^{l} e^{\mathrm{i}(m-n) \alpha} n !\left(x^{2}+y^{2}\right)^{\frac{m-n}{2}} L_{n}^{m-n}\left(x^{2}+y^{2}, \rho\right) \quad(\text { by }(\text { A.8 }))
\end{aligned}
$$


$=(-1)^{n} n !(x+\mathrm{i} y)^{m-n} L_{n}^{m-n}\left(x^{2}+y^{2}, \rho\right)$

(by polar coordinate transformation).

\section{A.3 Proof of Theorem 2.6}

\section{PROPOSITION A.6}

We have

$$
\partial J_{m, n}=m J_{m-1, n}, \quad \bar{\partial} J_{m, n}=n J_{m, n-1} .
$$

Proof

Since $\overline{\partial \phi}=\bar{\partial} \bar{\phi}$, we have $J_{m, n}(z, \rho)=\overline{J_{n, m}(z, \rho)}$. Thus we need only to prove that

$$
\partial J_{m, n}=m J_{m-1, n} .
$$

When $m=0$, both sides of (A.12) equal 0 . We proceed by induction on $m$. Assume that (A.12) is true for $m<k$. Then it follows from the commutation relation $\partial \partial^{*}-\partial^{*} \partial=\frac{1}{\rho}$ that

$$
\begin{aligned}
\partial J_{k, n} & =\partial\left(\rho \partial^{*} J_{k-1, n}\right)=\rho\left[\partial^{*} \partial J_{k-1, n}+\frac{1}{\rho} J_{k-1, n}\right] \\
& =\rho \partial^{*}(k-1) J_{k-2, n}+J_{k-1, n} \\
& =(k-1) J_{k-1, n}+J_{k-1, n} \\
& =k J_{k-1, n} .
\end{aligned}
$$

Proof of Theorem 2.5

We need only prove that

$$
J_{m, n}(z, \rho)=z^{m-n}(-1)^{n} n ! L_{n}^{m-n}\left(|z|^{2}, \rho\right), \quad m \geq n .
$$

The case $0=n \leq m$ is obvious. We proceed by induction on $m, n$. Assume that (A.13) is true for $n \leq l, m \leq k$.

Since $\partial^{*}$ commutes with $\bar{\partial}^{*}$, by the definition (2.8), we have

$$
\begin{aligned}
J_{m, l+1}(z, \rho) & =\rho \bar{\partial}^{*} J_{m, l}(z, \rho)=\bar{z} J_{m, l}(z, \rho)-\rho \partial J_{m, l}(z, \rho) \\
& =\bar{z} J_{m, l}(z, \rho)-m \rho J_{m-1, l}(z, \rho) \quad(\text { by }(\mathrm{A} .11)) \\
& =(-1)^{l} l ! z^{m-l-1}\left(|z|^{2} L_{l}^{m-l}\left(|z|^{2}, \rho\right)-m \rho L_{l}^{m-l-1}\left(|z|^{2}, \rho\right)\right) \\
& =(-1)^{l+1}(l+1) ! z^{m-l-1} L_{l+1}^{m-l-1}\left(|z|^{2}, \rho\right) \quad(\text { by }(\mathrm{A} .7)), \\
J_{k+1, n}(z, \rho) & =\rho \partial^{*} J_{k, n}(z, \rho)=z J_{k, n}(z, \rho)-\rho \bar{\partial} J_{k, n}(z, \rho) \\
& =z J_{k, n}(z, \rho)-n \rho J_{k, n-1}(z, \rho) \quad(\text { by }(\mathrm{A} .11)) \\
& =(-1)^{n} n ! z^{k+1-n}\left(L_{n}^{k-n}\left(|z|^{2}, \rho\right)-\rho L_{n-1}^{k-n+1}\left(|z|^{2}, \rho\right)\right) \\
& =(-1)^{n} n ! z^{k+1-n} L_{n}^{k+1-n}\left(|z|^{2}, \rho\right) \quad(\text { by }(\mathrm{A} .6)) .
\end{aligned}
$$


Proof of Theorem 2.6

Denote by $\langle f, g\rangle$ the inner product in the complex Hilbert space $L_{\mathbb{C}}^{2}(\mu)$.

(1) If $m-n \geq k-l \geq 0$, then

$$
\begin{aligned}
& \left\langle J_{m, n}, J_{k, l}\right\rangle \\
& \quad=(-1)^{n+l} \frac{n ! l !}{\pi \rho} \iint_{\mathbb{R}^{2}} z^{m-n} \bar{z}^{k-l} L_{n}^{m-n}\left(|z|^{2}, \rho\right) L_{l}^{k-l}\left(|z|^{2}, \rho\right) e^{-\frac{x^{2}+y^{2}}{\rho}} \mathrm{d} x \mathrm{~d} y
\end{aligned}
$$

(let $x=r \cos \theta, y=r \sin \theta$ and $s=r^{2}=x^{2}+y^{2}$ )

$$
\begin{aligned}
& =(-1)^{n+l} \frac{n ! l !}{2 \pi \rho} \int_{0}^{2 \pi} e^{\mathrm{i}[m-n-k+l] \theta} \mathrm{d} \theta \int_{0}^{\infty} s^{\frac{m-n+k-l}{2}} e^{-\frac{s}{\rho}} L_{n}^{m-n}(s, \rho) L_{l}^{k-l}(s, \rho) \mathrm{d} s \\
& =(-1)^{n+l} \frac{n ! l !}{\rho} \delta_{m-n, k-l} \int_{0}^{\infty} s^{m-n} e^{-\frac{s}{\rho}} L_{n}^{m-n}(s, \rho) L_{l}^{m-n}(s, \rho) \mathrm{d} s \\
& \left.=m ! n ! \rho^{m+n} \delta_{n l} \delta_{m-n, k-l} \quad \text { (by Proposition A.5 }(3)\right) .
\end{aligned}
$$

The other cases are similar.

This proves that the collection $\left\{\left(m ! n ! \rho^{m+n}\right)^{-\frac{1}{2}} J_{m, n}(z, \rho)\right\}$ is an orthonomal system. Therefore $\left\{J_{m, n}\right\}$ are linearly independent. It follows from (2.14) that they generate by linear combination the complex vector space of polynomials in terms of $z, \bar{z}$ (equal to in terms of $x, y \in \mathbb{R}$ ). It follows from [15, Lemma 2.4, p. 6] that the polynomials in the coordinate functions are dense in $L_{\mathbb{C}}^{2}(\mu)$. (Note that the lemma is still valid for the complex polynomials and the complex Hilbert space.) This proves that the orthonomal system is complete.

(2) Let $\alpha=m-n, u=|z|^{2}$. It follows from (A.11) that

$$
\frac{\partial^{2}}{\partial z \partial \bar{z}} J_{m, n}(z, \rho)=m n J_{m-1, n-1}
$$

and if $m>n$, then

$$
\begin{aligned}
& {\left[(1+\mathrm{i} c) z \frac{\partial}{\partial z}+(1-\mathrm{i} c) \bar{z} \frac{\partial}{\partial \bar{z}}-2 \rho \frac{\partial^{2}}{\partial z \partial \bar{z}}\right] J_{m, n}(z, \rho)} \\
& =(-1)^{n} n ! z^{m-n}\left[(1+\mathrm{i} c) m L_{n}^{\alpha-1}-(1-\mathrm{i} c) u L_{n-1}^{\alpha+1}+2 \rho m L_{n-1}^{\alpha}\right] \\
& =(-1)^{n} n ! z^{m-n} \\
& \quad \times\left[\left(2 m \rho L_{n-1}^{\alpha}+m L_{n}^{\alpha-1}+u \frac{\partial}{\partial u} L_{n}^{\alpha}\right)+\mathrm{i}\left(m L_{n}^{\alpha-1}-u \frac{\partial}{\partial u} L_{n}^{\alpha}\right) c\right] .
\end{aligned}
$$

Note that

$$
\begin{aligned}
m L_{n}^{\alpha-1}-u \frac{\partial}{\partial u} L_{n}^{\alpha} & =m L_{n}^{\alpha-1}-n L_{n}^{\alpha}+m \rho L_{n-1}^{\alpha} \\
& =m\left(L_{n}^{\alpha-1}+\rho L_{n-1}^{\alpha}\right)-n L_{n}^{\alpha}=(m-n) L_{n}^{\alpha}
\end{aligned}
$$

and

$$
\begin{aligned}
2 m \rho L_{n-1}^{\alpha}+m L_{n}^{\alpha-1}+u \frac{\partial}{\partial u} L_{n}^{\alpha} & =2 m \rho L_{n-1}^{\alpha}+m L_{n}^{\alpha-1}+n L_{n}^{\alpha}-m \rho L_{n-1}^{\alpha} \\
& =m\left(\rho L_{n-1}^{\alpha}+L_{n}^{\alpha-1}\right)+n L_{n}^{\alpha}=(m+n) L_{n}^{\alpha} .
\end{aligned}
$$


Substituting the above two equations into (A.15) yields (2.11) ${ }^{\dagger}$

(3) For the fixed $\lambda \in \mathbb{C}$, obviously the function $w(z)=\exp \left\{\lambda \bar{z}+\bar{\lambda} z-\rho|\lambda|^{2}\right\} \in$ $L_{\mathbb{C}}^{2}(\mu)$. Thus $w(z)$ has a unique series expression

$$
w(z)=\sum_{m=0}^{\infty} \sum_{n=0}^{\infty} a_{m, n} \frac{J_{m, n}(z, \rho)}{m ! n ! \rho^{m+n}},
$$

where the coefficients $a_{m, n}$ are given by

$$
\begin{aligned}
a_{m, n} & =\left\langle w, J_{m, n}\right\rangle=\rho^{m+n}\left\langle w,\left(\partial^{*}\right)^{m}\left(\bar{\partial}^{*}\right)^{n} 1\right\rangle \\
& =\rho^{m+n}\left\langle\partial^{m} \bar{\partial}^{n} w, 1\right\rangle=\bar{\lambda}^{m} \lambda^{n} \rho^{m+n}\langle w, 1\rangle \\
& =\bar{\lambda}^{m} \lambda^{n} \rho^{m+n} .
\end{aligned}
$$

Substituting it into (A.16) yields (2.12). In addition, by the well-known classical global uniform estimates given by Szegö [21], $\left|L_{n}^{\alpha}(x)\right| \leq \frac{(\alpha+1)_{n}}{n !} e^{\frac{x}{2}}, \alpha, x \geq 0$, one can show that the convergence is absolute and uniform on compact sets in $(\lambda, z)$. Thus the convergence is also pointwise, and the equality holds everywhere. ${ }^{\dagger \dagger}$

\section{A.4 Itô's complex multiple Wiener integral}

For the reader's convenience, we summarize Itô's work on the complex multiple Wiener integral (see [8]). By analogy with the relation between the real multiple Itô-Wiener integral and Hermite polynomials (see [7], [11])

$$
H_{n}\left(\xi_{t}\right)=\int_{0}^{t} \int_{0}^{t} \cdots \int_{0}^{t} \mathrm{~d} \xi_{t_{1}} \cdots \mathrm{d} \xi_{t_{n}}
$$

where $H_{n}$ denotes the $n$th Hermite polynomial with leading coefficient 1 . K. Itô obtained the relation between Hermite-Laguerre-Itô polynomials and the complex multiple Itô-Wiener integral.

If $\left(B_{1}, B_{2}\right)$ denotes 2-dimensional Brownian motion, we put $\zeta_{t}:=B_{1}(t)+$ $\mathrm{i} B_{2}(t)$ with $\mathrm{i}=\sqrt{-1} ; \zeta_{t}$ is called complex Brownian motion. Let $\bar{\zeta}_{t}$ be the complex conjugate of $\zeta_{t}$.

\section{NOTATION 1}

For $m, n \in \mathbb{N}$, denote $F_{m, n}\left(\zeta_{t}\right)=\frac{(-1)}{m ! n !} J_{m, n}\left(\zeta_{t}, \mathbb{E}\left|\zeta_{t}\right|^{2}\right)$.

By the formula for integration by parts (stochastic product rule) and Itô's formula, we have the following.

PROPOSITION A.7

$F_{m, n}\left(\zeta_{t}\right), m, n \in \mathbb{N}$ satisfy

$$
\mathrm{d} F_{m, n}\left(\zeta_{t}\right)=F_{m-1, n}\left(\zeta_{t}\right) \mathrm{d} \zeta_{t}+F_{m, n-1}\left(\zeta_{t}\right) \mathrm{d} \bar{\zeta}_{t}
$$

\footnotetext{
$\dagger$ There is a direct way to prove (2.11) by using [8, Theorem 12(E)]. To be self-contained, here we use the equalities of Laguerre polynomials.

${ }^{\dagger \dagger}$ K. Itô showed (2.12) by means of power series expansion (see [8]).
} 
By iteration, we have the following.

\section{COROLLARY A. 8}

$F_{m, n}\left(\zeta_{t}\right)$ can be decomposed into the iterated Itô integrals of complex Brownian motion as

$$
F_{m, n}\left(\zeta_{t}\right)=\sum \int_{0}^{t} \int_{0}^{t_{m+n}} \cdots \int_{0}^{t_{2}} \mathrm{~d} C_{t_{1}} \mathrm{~d} C_{t_{2}} \cdots \mathrm{d} C_{t_{m+n}},
$$

where $0<t_{1}<t_{2}<\cdots<t_{m+n}<t, C_{t}=\zeta_{t}$ or $C_{t}=\bar{\zeta}_{t}$, and the sum is over all the $n$-combinations of $\{1,2, \ldots, m+n\}$ such that $C_{t}=\bar{\zeta}_{t}$.

Using the approximation by off-diagonal step functions (i.e., the analogue of the multiple Itô-Wiener integral [11, Definition 9.6.5]),

$$
\begin{aligned}
& \int_{0}^{t} \int_{0}^{t} \cdots \int_{0}^{t} \mathrm{~d} \zeta_{t_{1}} \cdots \mathrm{d} \zeta_{t_{m}} \mathrm{~d} \bar{\zeta}_{t_{m+1}} \cdots \mathrm{d} \bar{\zeta}_{t_{m+n}} \\
& \quad=m ! n ! \sum \int_{0}^{t} \int_{0}^{t_{m+n}} \cdots \int_{0}^{t_{2}} \mathrm{~d} C_{t_{1}} \mathrm{~d} C_{t_{2}} \cdots \mathrm{d} C_{t_{m+n}}
\end{aligned}
$$

which can be seen as a generalization of [11, Theorem 9.6.7] to complex Brownian motion. Thus, we have the following.

\section{COROLLARY A.9}

$J_{m, n}\left(\zeta_{t}, \mathbb{E}\left|\zeta_{t}\right|^{2}\right)$ is related to the complex multiple Itô-Wiener integral,

$$
J_{m, n}\left(\zeta_{t}, \mathbb{E}\left|\zeta_{t}\right|^{2}\right)=\int_{0}^{t} \int_{0}^{t} \cdots \int_{0}^{t} \mathrm{~d} \zeta_{t_{1}} \cdots \mathrm{d} \zeta_{t_{m}} \mathrm{~d} \bar{\zeta}_{t_{m+1}} \cdots \mathrm{d} \bar{\zeta}_{t_{m+n}}
$$

\section{References}

[1] R. Balescu, Statistical Dynamics: Matter out of Equilibrium, Imperial College Press, London, 1997.

[2] A. Chojnowska-Michalik and B. Goldys, Nonsymmetric Ornstein-Uhlenbeck semigroup as second quantized operator, J. Math. Kyoto Univ. 36 (1996), 481-498. MR 1417822.

[3] Symmetric Ornstein-Uhlenbeck semigroups and their generators, Probab. Theory Related Fields 124 (2002), 459-486. MR 1942319. DOI 10.1007/s004400200222.

[4] R. A. Conway, A Course in Functional Analysis, 2nd ed., Grad. Texts in Math. 96, Springer, New York, 1990. MR 1070713.

[5] R. Courant and D. Hilbert, Methods of Mathematical Physics, Vol. 1, Interscience, New York, 1966. MR 0195654.

[6] R. A. Horn and C. R. Johnson, Matrix Analysis, Cambridge Univ. Press, Cambridge, 1985. MR 0832183. 
[7] K. Itô, Multiple Wiener integral, J. Math. Soc. Japan 3 (1951), 157-169. MR 0044064.

[8] Complex multiple Wiener integral, Japan J. Math. 22 (1953), 63-86. MR 0063609.

[9] S. Janson, Gaussian Hilbert Spaces, Cambridge Tracts Math. 129, Cambridge Univ. Press, Cambridge, 2008. MR 1474726. DOI 10.1017/CBO9780511526169.

[10] R. V. Kozhan, $L^{1}$-spectrum of Banach space valued Ornstein-Uhlenbeck operators, Semigroup Forum 78 (2009), 547-553. MR 2511784.

DOI 10.1007/s00233-008-9088-y.

[11] H.-H. Kuo, Introduction to Stochastic Integration, Universitext, Springer, New York, 2006. MR 2180429.

[12] S. Kusuoka and I. Shigekawa, Exponential convergence of Markovian semigroups and their spectra on $L^{p}$-spaces, preprint, 2012.

[13] N. N. Lebedev, Special Functions and Their Applications, rev. ed., Dover, New York, 1972. MR 0350075.

[14] A. Lunardi, On the Ornstein-Uhlenbeck operator in $L^{2}$ spaces with respect to invariant measures, Trans. Amer. Math. Soc. 349 (1997), no. 1, 155-169.

MR 1389786. DOI 10.1090/S0002-9947-97-01802-3.

[15] P. Malliavin, Stochasitc Analysis, Grund. Math. Wiss. 313, Springer, Berlin, 1997. MR 1450093.

[16] G. Metafune and D. Pallara, Spectrum of Ornstein-Uhlenbeck operators in $L^{p}$ spaces with respect to invariant measures, J. Funct. Anal. 196 (2002), 40-60.

MR 1941990. DOI 10.1006/jfan.2002.3978.

[17] S. Peszat, Lévy-Ornstein-Uhlenbeck transition semigroup as second quantized operator, J. Funct. Anal. 260 (2011), 3457-3473. MR 2781967.

DOI 10.1016/j.jfa.2011.03.002.

[18] I. V. Proskuryakov, Problems in Linear Algebra, Victor Kamkin, Moscow, 1978. MR 0524396.

[19] M. Reed and B. Simon, Methods of Modern Mathematical Physics, I: Functional Analysis, 2nd ed., Academic, New York, 1980. MR 0751959.

[20] I. Shigekawa, Stochastic Analysis, Trans. Math. Monogr. 224, Iwanami Ser. Modern Math., Amer. Math. Soc., Providence, 2004. MR 2060917.

[21] G. Szegö, Orthogonal Polynomials, 4th ed., Amer. Math. Soc. Colloq. Publ. 23, Amer. Math. Soc., Providence, 1975. MR 0372517.

[22] J. van Neerven, Nonsymmetric Ornstein-Uhlenbeck semigroups in Banach spaces, J. Funct. Anal. 155 (1998), 495-535. MR 1624573.

DOI 10.1006/jfan.1997.3237.

[23] Second quantization and the $L^{p}$-spectrum of nonsymmetric Ornstein-Uhlenbeck operators, Infin. Dimens. Anal. Quantum. Probab. Relat. Top. 8 (2005), 473-495. MR 2172310. DOI 10.1142/S0219025705002074.

[24] Z. X. Wang and D. R. Guo, Special Functions, World Sci., Teaneck, N.J., 1989. MR 1034956. 
Chen: School of Mathematics and Computing Science, Hunan University of Science and Technology, Xiangtan, Hunan, 411201, P.R. China; chenyong77@gmail.com

Liu: LMAM, School of Mathematical Sciences, Center for Statistical Science, Peking University, Beijing, 100871, P.R. China; liuyong@math.pku.edu.cn 